\title{
Viscoelastic properties measurements of thin polymer films from reflow of nanoimprinted patterns
}

\author{
Etienne Rognin a) and Stefan Landis \\ CEA-LETI-Minatec Campus, 17 Rue des Martyrs, 38054 Grenoble Cedex 9, France \\ Laurent Davoust \\ SIMAP/EPM, Grenoble Institute of Technology, 1130 Rue de la Piscine, 38400 Saint Martin d'Hères, France
}

(Received 27 June 2011; accepted 5 November 2011; published 15 December 2011)

\begin{abstract}
The authors describe in this paper a fast and cost-effective method to measure the viscoelastic properties of a thin polymer film from the reflow of nanoimprinted patterns. The material is spin-coated onto a silicon substrate and specially designed nanopatterns are imprinted on the film using thermal nanoimprint. A first measurement of the imprinted profile is done by atomic force microscopy (AFM). The film is then heated at a definite temperature above the glass transition temperature during a definite time. The film is rapidly cooled down and the reflowed profile is again measured by AFM. Spectral densities of the profiles are computed using standard Fourier transform algorithms, and the viscoelastic properties are computed as fitting parameters of an evolution model for the spectral density of the topology. The originality of our method is based on the accurate spatial description of the imprint rather than on its temporal decay. Using our approach, we measured the viscoelastic properties of a $205 \mathrm{~nm}$-thick polystyrene (molecular weight $130 \mathrm{~kg} / \mathrm{mol}$ ) film, assuming a single relaxation time Maxwell model. (C) 2012 American Vacuum Society. [DOI: 10.1116/1.3664088]
\end{abstract}

\section{INTRODUCTION}

NanoImprint Lithography (NIL) emerged in the mid$1990 \mathrm{~s}^{1}$ and has been rapidly considered as a high resolution and high throughput patterning technique. Despite a huge development of imprinting equipments, stamp manufacturing processes, imprint processes, dedicated materials, and metrology approaches, a complete simulation toolbox of NIL is still lacking. Characterization of flow properties of melted polymer at macroscopic scale is now quite an easy task to perform from classical measurement devices devoted to volume rheology. However, in NIL processes, resist film thickness is ranging from several tens of nanometer up to several hundreds of nanometer. In such configuration, surface phenomena play a decisive role with significant deviation for viscous flows properties. ${ }^{2}$

Existing methods to measure the viscoelastic properties of fluids at nanoscopic scale are usually based on the nucleation and growth rate of holes in a dewetting polymer film, 3,4 atomic force microscopy (AFM) probing, ${ }^{5-7}$ or relaxation dynamics of a surface relief. In the latter case, we can distinguish between methods where the topography is either induced by thermal fluctuations, ${ }^{8,9}$ or imprinted by a mold, using common nanoimprint techniques. ${ }^{10-14}$ The dynamics is probed using $x$ ray photon correlation microscopy, or more generally by light scattering techniques. In this approach, attention is given to the time evolution of the fundamental spatial frequency of the shape, and viscoelastic properties are determined from the measured relaxation time. In many cases, neither the exact topology nor the thermal dependence of the optical index of the material are known, and a delicate data processing is needed.

$\overline{{ }^{a} \text { Electronic mail: etienne.rognin@cea.fr }}$
In this paper, we present a method to measure the viscoelastic properties of a thin polymer film from the reflow of nanoimprinted patterns. We focus our efforts on the accurate spatial determination of the film surface, rather than on its temporal evolution, and we extend the method formulated by Leveder et al. ${ }^{15,16}$ AFM measurements with a spatial resolution lower than $1 \mathrm{~nm}$ are used to extract the spectral density of the topography at two different times of the reflow. The mechanical properties of the film are extracted from the comparison of these two densities, with a robust analytical model. The imprinted patterns are specially designed to provide relevant spectral densities, in order to perform measurements in a large range of mechanical properties and temperatures.

\section{METHOD}

In this section, we present the main steps of the method, drawn in Fig. 1. The material (polymer) is spin-coated onto a silicon substrate (a) and the film thickness is measured by ellipsometry. We begin with imprinting the film by thermal nanoimprint $^{1}(\mathrm{~b})$, using a specially designed mold which will be described hereafter. After demolding at room temperature, a first measurement of the imprinted profile is done by AFM (c). The film is then heated at a definite temperature above the glass transition temperature $\left(T_{g}\right)$ during a definite time (d). The film is then rapidly cooled down and the reflowed profile is again measured by AFM (e). Spectral densities of the profiles are computed using standard Fourier transform algorithms. The viscoelastic properties are fitted from the spectral density evolution of the topology, described in the next section.

At temperature above $T_{g}$, the film flows under surface ten$\operatorname{sion}^{15,17,18}$ and reaches a steady state when the surface energy of the free interface is minimum; in other words when the film is completely flat. During the reflow process, 
(a) Material coating

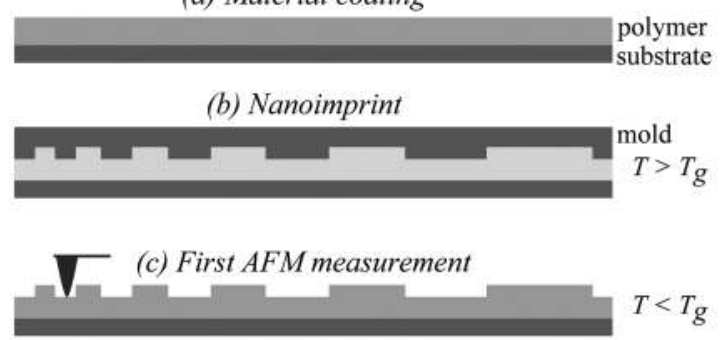

(d) Reflow

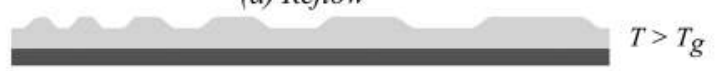

(e) Second AFM measurement
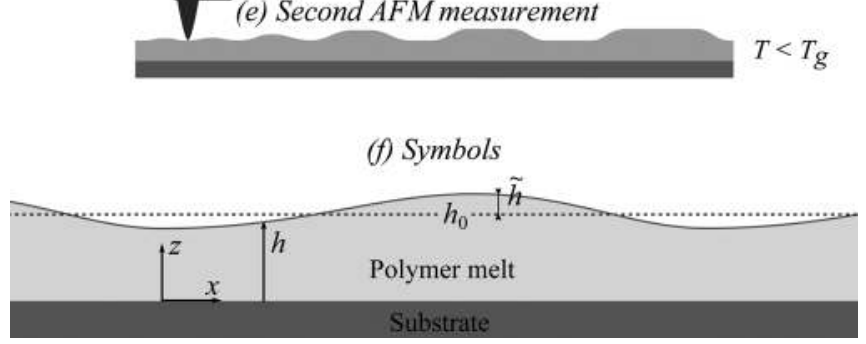

FIG. 1. Main steps of the viscoelasticity measurement method: (a) Material coating. (b) Nanoimprint. (c) First AFM measurement. (d) Reflow. (e) Second AFM measurement. (f) Symbols use in the model description.

high frequency features tend to disappear faster than general shapes, resulting in the smoothing of the patterns, which has been observed in numerous experimental works. ${ }^{19-21}$ The decay rate of each mode is given by the mechanical properties of the film. The measurement of this spatial filtering is then used to compute the viscoelastic properties of the material.

\section{MODEL}

We assume a two-dimensional flow [Fig. 1(f)] described by the laws of continuum mechanics. The film thickness is the local and instantaneous function $h(x, t)$, written: $h=h_{0}+\tilde{h}(x, t)$, where $h_{0}$ is the mean thickness of the film (over time and space). In order to derive a simple analytical model for the flow, we assume that the deviation of the free surface from the mean thickness value is small; in other words that $\tilde{h} / h_{0} \ll 1$. The standard boundary conditions are: no slip at the bottom interface and surface tension at the top interface. Here we neglect the disjoining pressure. ${ }^{22}$

This kind of microfluidics system was previously solved by Henle and Levine ${ }^{23}$ for the characterization of spontaneous capillary waves, using a Fourier analysis. With the Fourier transform $h(x) \rightarrow \hat{h}(k)$, the evolution model for the spectral density is

$$
\hat{h}(k, t)=\hat{h}(k, 0) \times \exp \left(-\frac{t}{\tau(k)}\right),
$$

where $t$ is the duration of the reflow and $\tau(k)$ is the decay rate of the mode $k$. The decay rate $\tau=1 / i \omega$ is given by a dispersion relation computed from the mechanical equations and the boundary conditions

$$
\frac{h_{0} G(\omega)}{\gamma}+f\left(k h_{0}\right)=0,
$$

where $\gamma$ is the surface tension, $G(\omega)$ is the complex shear modulus, ${ }^{24}$ and $f$ is a dimensionless function of the normalized wave vector $k h_{0}$, given by

$$
f\left(k h_{0}\right)=\frac{\sinh k h_{0} \cosh k h_{0}-k h_{0}}{2 k h_{0} \cosh ^{2} k h_{0}} k^{2} h_{0}^{2} .
$$

For a Maxwell fluid with a single mode, $G(\omega)=-i \omega \eta$ $/\left(1-i \omega \tau_{x}\right)$, where $\eta$ is the viscosity at slow strain and $\tau_{x}$ is the relaxation time. Note that at rapid strain, the material behaves like an elastic solid of shear elasticity $G=\eta / \tau_{x}$. From the dispersion relation we get

$$
\tau(k)=\frac{\eta h_{0}}{\gamma f\left(k h_{0}\right)}+\tau_{x}
$$

Finally, Eqs. (1) and (4) give the theoretical damping function

$$
\left.\frac{\hat{h}(k, t)}{\hat{h}(k, 0)}=\exp -\frac{1}{\mathrm{Ca} \times f\left(k h_{0}\right)^{-1}+\mathrm{De}}\right),
$$

where the dimensionless parameters are the capillary number $\mathrm{Ca}=\eta h_{0} / \gamma t$ and the Deborah number De $=\tau_{x} / t$. The theoretical function of Eq. (5) can be fitted to the experimental damping factors $\hat{h}(k, t) / \hat{h}(k, 0)$, and the two parameters $\eta$ and $\tau_{x}$ can then be extracted.

\section{MOLD}

From the previous section and especially from Eq. (1), we see that there are two ways to extract the mechanical properties of the film, by measuring either the exponential temporal decay of a single mode, ${ }^{8-14}$ or the damping factor for each mode at a given time. Since we focus on the second method, in order to ensure relevant data, the spectral density of the imprinted profile has to be as rich as possible, but keeping the profile short enough to be measured by a single AFM scan. We present below a patented approach to designing a mold with such a profile.

We can define a pattern shape $m(x)$ of length $L$, of depth $2 \tilde{m}$ by the modulation function

$$
m(x)=\tilde{m} \times \operatorname{sign}\left[\cos \left(\int_{0}^{x} k(\xi) \mathrm{d} \xi\right)\right],
$$

where $k(\xi)$ is the local wave vector. Note that if $k(\xi)=k_{1}$ (the pattern is periodical), then $m(x)=\tilde{m} \times \operatorname{sign}\left[\cos k_{1} x\right]$. The choice of the function $k(\xi)$ is essential to determine the spectral density. A uniform spectral density (same weight given to low and high frequencies) is achieved by

$$
k(x)=k_{\max }+\left(k_{\min }-k_{\max }\right) \times \frac{x}{L},
$$

where $k_{\min }$ and $k_{\max }$ are, respectively, the minimum and the maximum local wave vectors. This function $k(\xi)$ is relevant 
for AFM scans, but other functions could be selected depending on the measurement equipment.

The next section presents an implementation of the method to measure the mechanical properties of a polymer thin film

\section{EXPERIMENTS AND DISCUSSION}

Low molecular weight $\left(M_{w}=130 \mathrm{~kg} \cdot \mathrm{mol}^{-1}\right)$ monodisperse $\left(M_{w} / M_{n}<1.06\right)$ polystyrene (PS $\left.130 \mathrm{k}\right)$ solution is spin-coated onto 8 in. silicon substrates. Prior to coating PS, an $\mathrm{O}_{2} / \mathrm{N}_{2} \mathrm{H}_{2}(2150 \mathrm{sccm} / 240 \mathrm{sccm})$ plasma is performed during $180 \mathrm{~s}$ at $543 \mathrm{~K}$ in order to obtain a homogeneous $4 \mathrm{~nm}$ thick $\mathrm{SiO}_{\mathrm{x}}$ layer. The glass transition temperature $\left(T_{g}=102 \pm 1{ }^{\circ} \mathrm{C}\right)$ is measured by differential scanning calorimetry (DSC). The value of the surface tension is given in the literature ${ }^{24}$ and is $\gamma=32 \pm 2 \mathrm{mN} \cdot \mathrm{m}^{-1}$. The thicknesses of the coated polystyrene films were measured by ellipsometry and were $h_{0}=205 \pm 1 \mathrm{~nm}$. The imprints of the patterns were made at 13 bars and at temperature $T_{g}+80^{\circ} \mathrm{C}$ where the elastic stress relaxes quickly (measured by disk rheometry to be less than $1 \mathrm{~s}$ ) in comparison to the imprint time (which was at least $10 \mathrm{~min}$ ). The mold was manufactured in CEALETI clean room using electron-beam lithography and silicon etching processes. This $8 \mathrm{in}$. silicon stamp was coated with an anti-sticking layer. ${ }^{25} \mathrm{~A}$ constant residual layer is achieved thanks to the small depth of the pattern $(70 \mathrm{~nm})$, and to the small length of the pattern $(40 \mu \mathrm{m})$ compared to the thickness of the mold $(750 \mu \mathrm{m})$. Demolding was performed at room temperature. Figure 2 shows an AFM image of the imprinted pattern just after demolding. The residual layer is $170 \mathrm{~nm}$, and the pitch of the lines ranges from $416 \mathrm{~nm}$ to $4.5 \mu \mathrm{m}$.

Figure 3 gives an example of a reflow experiment. The PS $130 \mathrm{k}$ film was annealed at $140{ }^{\circ} \mathrm{C}$ during $t=30 \mathrm{~s}$. The low-pass filtering behavior of the reflow is clearly confirmed on Fig. 3, both by the AFM profiles and the spectral densities where high order modes disappeared after annealing.

The damping factor $\hat{h}(k, t) / \hat{h}(k, 0)$ is computed from the spectral densities, and the parameters of the damping model [Eq. (5)] are fitted to the experimental points (Fig. 4). The experimental result of Fig. 4 shows that a viscoelastic Maxwell model is more relevant to describe the flow than a purely viscous model, especially for high spatial frequencies. The result of the fitting is $\mathrm{Ca}=0.99 \pm 0.20$ and $\mathrm{De}=0.36$ \pm 0.086 . The final parameters are $\eta=5.7 \pm 1.2 \times 10^{6} \mathrm{Pas}$ and $\tau_{x}=10 \pm 2.4 \mathrm{~s}$.

The method was used to measure the viscoelastic properties of PS $130 \mathrm{k}$ at various temperatures. Results are reported

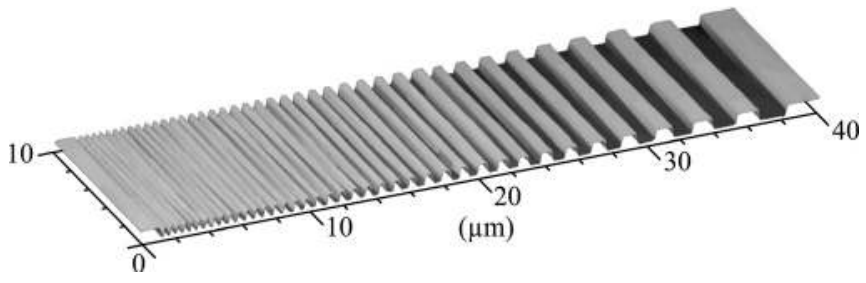

FIG. 2. AFM images of an imprinted pattern just after demolding. The imprinted depth was $70 \mathrm{~nm}$. (a) AFM measurements

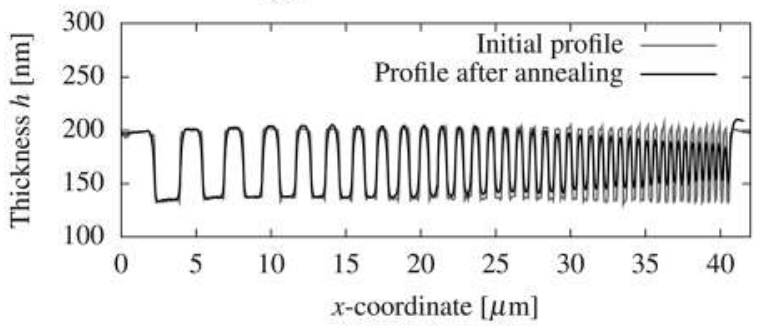

(b) Spectral densities

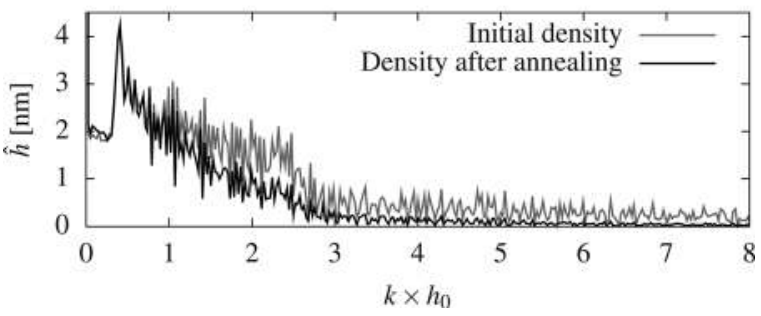

FIG. 3. Single temperature measurement example. Polystyrene $\left(M_{w}=130\right.$ $\mathrm{kg} \cdot \mathrm{mol}^{-1}$ ) was annealed at $140^{\circ} \mathrm{C}$ during $30 \mathrm{~s}$. (a) AFM Profiles of the imprinted profile before and after annealing. (b) Fourier transforms of the AFM profiles.

in Fig. 5. Both the viscosity [Fig. 5(a)] and the relaxation time [Fig. 5(b)] exhibit an exponential decrease as temperature increases, which is consistent with the time-temperature equivalence. ${ }^{26} \mathrm{~A}$ specific comparison with bulk rheology based upon the work of Majeste $e t$ al. ${ }^{27}$ can be made. The authors studied the viscoelastic properties of polystyrene samples of numerous molecular weights. They extracted the parameters of the Vogel model giving the viscosity $\eta$ of polystyrene of molecular weight $M_{w}$ as a function of temperature:

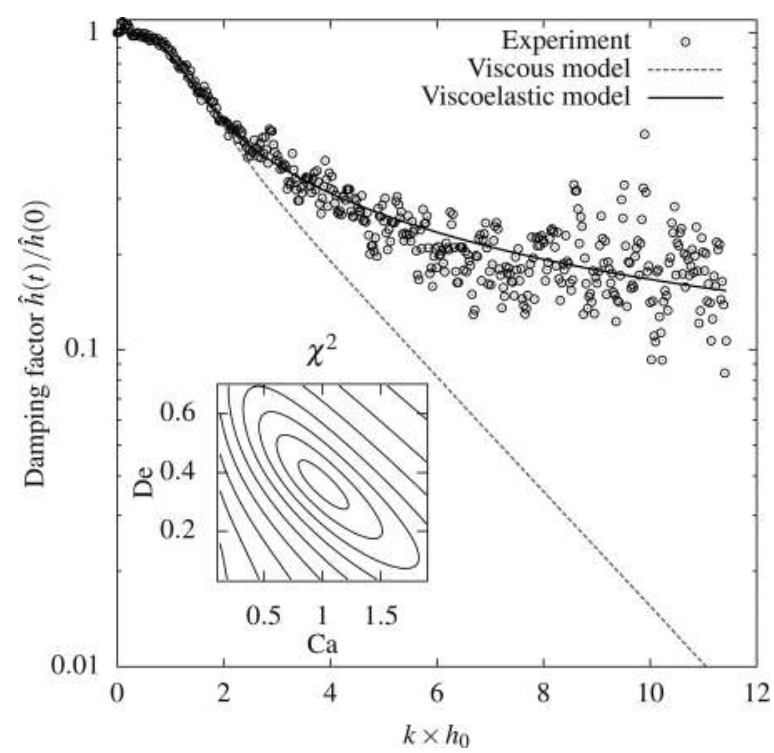

FIG. 4. Damping factor $\hat{h}(t) / \hat{h}(0)$ as a function of the normalized wave vector. Experimental points are fitted with two models: a purely viscous model $\left(\tau_{x}=0\right)$ and a viscoelastic single mode Maxwell model. Inset: arbitrary levels of the cost function $\chi^{2}$ (least squares fitting of the Maxwell model) showing a single minimum. The fitted parameters are $\mathrm{Ca}=0.99 \pm 0.20$ and $\mathrm{De}=0.36 \pm 0.086$ 
(a) Viscosity

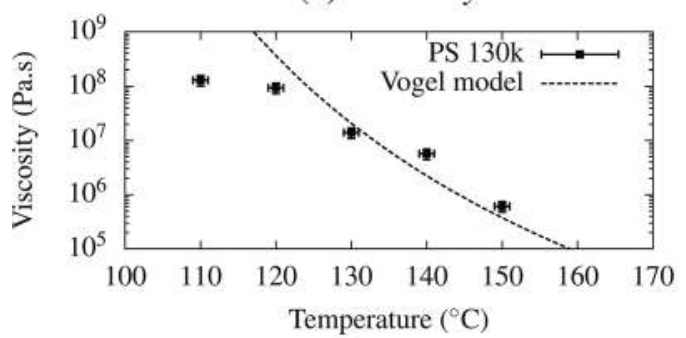

(b) Elastic properties

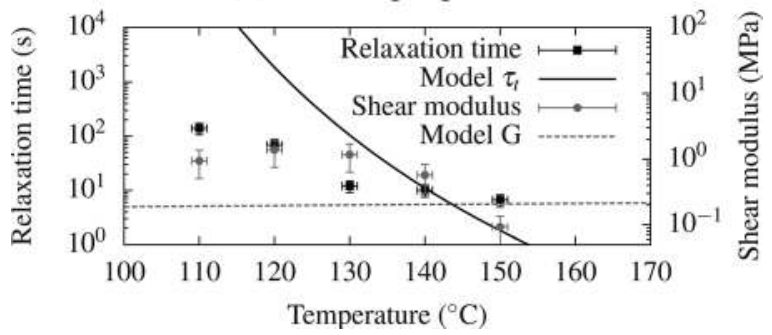

FIG. 5. Results for PS $130 \mathrm{k}$ at several temperatures. (a) Experimental viscosity (points) and the Vogel model (dashed line). (b) Elastic properties. Left axis: experimental relaxation time (black points) and model (black line). Right axis: experimental shear modulus (gray points) and model (gray line).

$$
\eta\left(M_{w}, T\right)=A_{\text {ref }} \exp \left(\frac{B}{\alpha_{f}\left(T-T_{\text {ळref }}\right)}\right) \times\left(\frac{M_{w}}{M_{\text {ref }}}\right)^{3.4},
$$

where $A_{\text {ref }}, B, \alpha_{f}$, and $T_{\infty \text { ref }}$ are the parameters of the model for a given referenced molecular weight $M_{\text {ref }}$. The exponent 3.4 accounts for the dependence of viscosity on molecular mass above the critical mass $M_{c} \approx 35 \mathrm{~kg} \cdot \mathrm{mol}^{-1}$. Table I in Ref. 27 gives for $M_{\text {ref }}=110 \mathrm{~kg} \cdot \mathrm{mol}^{-1}: A_{\text {ref }}=3.691$ $\times 10^{-2} \mathrm{Pas}$, and $T_{\infty \mathrm{ref}}=49.6^{\circ} \mathrm{C}$. The parameter $B / \alpha_{f}$ does not depend on mass, and is $B / \alpha_{f}=1620 \pm 50 \mathrm{~K}$. The model curve (8) is plotted on Fig. 5(a) and reasonably accounts for experimental data above $120^{\circ} \mathrm{C}$. We can also estimate the shear modulus $G=\eta / \tau_{t}$ associated with the terminal relaxation time $\tau_{t}$ of polystyrene in the entangled regime, given by the following equation:

$$
G(T)=\frac{\rho\left(M_{w}, T\right) R T}{M_{e}},
$$

where $\rho\left(M_{w}, T\right)$ is the mass density of polystyrene [given by formula (19) in Ref. 27], $R$ is the gas constant, and $M_{e} \approx 17 \mathrm{~kg} \cdot \mathrm{mol}^{-1}$ is the entanglement molecular mass. Curve (9) is plotted on Fig. 5(b). Although the computed modulus does not exactly fit with the experimental values, it gives a consistent order of magnitude and accounts for the low dependence of the experimental shear modulus on temperature.

The terminal time $\tau_{t}(T)=\eta(T) / G(T)$ is also plotted on Fig. 5(b). The large discrepancy with experimental data for low temperatures suggests that the observed relaxation time at these temperatures may not be the terminal time but a relaxation time (or a combination of times) associated to faster dynamics. $^{27}$

\section{CONCLUSION}

In this paper, we presented a method to measure the viscoelastic properties of polymer thin films from the reflow of nanoimprinted patterns. We derived a complete and accurate model for the spectral density evolution of the topology. Contrary to previous similar approaches, emphasis was put on the spatial description rather than the temporal decay of the patterns. With this model, we could develop relevant stamp designs with spatially modulated patterns to optimize experimental measurements and to ensure fruitful data processing. Our approach was then successfully applied to measure the viscoelastic properties of PS $130 \mathrm{k}$. Our method does not require a dedicated equipment since it makes use of imprint tools and AFM. It is then a fast and cost-effective method to measure the rheologic properties of polymer thin films.

${ }^{1}$ S. Y. Chou, P. R. Krauss, and P. J. Renstrom, Appl. Phys. Lett. 67, 3114 (1995).

${ }^{2}$ H. Bodiguel and C. Fretigny, Phys. Rev. Lett. 97, 266105 (2006).

${ }^{3}$ H. Bodiguel and C. Fretigny, Eur. Phys. J. E 19, 185 (2006).

${ }^{4}$ S. Gabriele, P. Damman, S. Sclavons, S. Desprez, S. Coppée, G. Reiter, M. Hamieh, S. A. Akhrass, T. Vilmin, and E. Raphaël, J. Polym. Sci., Part B: Polym. Phys. 44, 3022 (2006).

${ }^{5}$ P. Attard, J. Phys. Condens. Matter 19, 473201 (2007).

${ }^{6}$ G. Moeller, J. Polym. Sci., Part B: Polym. Phys. 47, 1573 (2009).

${ }^{7} \mathrm{X}$. Zhang, X. Zhang, and T. Chen, in Proceedings of the Society of Tribologists and Lubrication Engineers, 2010 (unpublished), pp. 652-654.

${ }^{8}$ H. Kim, A. Rühm, L. B. Lurio, J. K. Basu, J. Lal, D. Lumma, S. G. J. Mochrie, and S. K. Sinha, Phys. Rev. Lett. 90, 068302 (2003).

${ }^{9}$ Z. Jiang, H. Kim, X. Jiao, H. Lee, Y. Lee, Y. Byun, S. Song, D. Eom, C. Li, M. H. Rafailovich, L. B. Lurio, and S. K. Sinha, Phys. Rev. Lett. 98, 227801 (2007)

${ }^{10}$ M. Hamdorf and D. Johannsmann, J. Chem. Phys. 112, 4262 (2000).

${ }^{11}$ R. L. Jones, T. Hu, C. L. Soles, E. K. Lin, R. M. Reano, S.W. Pang, and D. M. Casa, Nano Lett. 6, 1723 (2006).

${ }^{12}$ Y. Ding, H. W. Ro, J. F. Douglas, R. L. Jones, D. R. Hine, A. Karim, and C. L. Soles, Adv. Mater. 19, 1377 (2007).

${ }^{13}$ Y. Ding, H. W. Ro, T. A. Germer, J. F. Douglas, B. C. Okerberg, A. Karim, and C. L. Soles, ACS Nano 1, 84 (2007).

${ }^{14}$ Y. Ding, H. W. Ro, K. J. Alvine, B. C. Okerberg, J. Zhou, J. F. Douglas, A. Karim, and C. L. Soles, Adv. Funct. Mater. 18, 1854 (2008).

${ }^{15}$ T. Leveder, S. Landis, and L. Davoust, Appl. Phys. Lett. 92, 013107 (2008).

${ }^{16}$ T. Leveder, S. Landis, N. Chaix, and L. Davoust, J. Vac. Sci. Technol. B 28, 1251 (2010).

${ }^{17}$ A. Oron, S. H. Davis, and S. G. Bankoff, Rev. Mod. Phys. 69, 931 (1997).

${ }^{18}$ K. J. Alvine, Y. Ding, J. F. Douglas, H. W. Ro, B. C. Okerberg, A. Karim, and C. L. Soles, Soft Matter 5, 2913 (2009).

${ }^{19}$ Z. Yu, L. Chen, W. Wu, H. Ge, and S. Y. Chou, J. Vac. Sci. Technol. B 21, 2089 (2003).

${ }^{20}$ C. Chao and L. J. Guo, Appl. Phys. Lett. 84, 2479 (2004).

${ }^{21}$ S. Y. Chou and Q. Xia, Nat. Nanotechnol. 3, 295 (2008).

${ }^{22} \mathrm{~J}$. N. Israelachvili, Intermolecular and Surface Forces, 2nd ed. (Academic, New York, 1991).

${ }^{23}$ M. L. Henle and A. J. Levine, Phys. Rev. E 75, 021604 (2007).

${ }^{24} \mathrm{~J}$. Bicerano, Prediction of Polymer Properties (Marcel Dekker, New York, 2002).

${ }^{25}$ S. Landis, N. Chaix, C. Gourgon, and T. Leveder, Nanotechnol. 19, 125305 (2008).

${ }^{26}$ M. L. Williams, R. F. Landel, and J. D. Ferry, J. Am. Chem. Soc.77, 3701 (1955).

${ }^{27}$ J. Majeste, J. Montfort, A. Allal, and G. Marin, Rheol. Acta 37, 486 (1998). 